\title{
Ideen gibt es genug: Budgetcafé, Budgetbegleiter, Budgetbüro ...
}

Bei der rechtsverbindlichen Einführung des Persönlichen Budgets zum Januar 2008 wurde immer wieder ein Paradigmenwechsel beschworen, durch den das bislang vorherrschende, statische Leistungsdreieck zwischen Leistungsträger, Leistungsempfänger und Leistungserbringer aufgelöst werden soll. Der Mensch mit Behinderung wird den Intentionen dieses Budgetkonzepts zufolge in seinem Leistungsbezug als Kunde und Arbeitgeber wahrgenommen, der über seine Hilfe- und Unterstützungsleistungen selbst bestimmen kann. Die Komplexität der Antragsstellung, das Nachweisen und Durchfechten des jeweiligen Leistungsbedarfs gegenüber den zuständigen Behörden stellt jedoch vor allem für Menschen mit einer sogenannten geistigen Behinderung eine große Hürde dar.

Ob sich eine soziale Organisation dafür entscheidet, die Einführung des Persönlichen Budgets mit entsprechenden Maßnahmen von Anfang an zu fördern, liegt natürlich in der Verantwortung der jeweiligen Organisation. Der Martinsclub Bremen e. V. hat sich in einer Grundsatzentscheidung unter der Beteiligung von Mitarbeiterinnen und Mitarbeitern seiner unterschiedlichen Fachbereiche (Wohnen, Bildung und Freizeit, Arbeit und Qualifizierung, Assistenz in Schulen) dafür entschieden, das Persönliche Budget mit allen dem Verein zur Verfügung stehenden Mitteln zu unterstützen. Diese Haltung beinhaltet die Bereitschaft, sich mit einer ansteigenden Zahl von Budgetnehmerinnen und Budgetnehmern einem konkurrierendem Markt zu öffnen und vorhandene Angebote umzustrukturieren und auch gänzlich neue Angebote zu entwickeln.

Um das Persönliche Budget aktiv mit Leben zu füllen, muss neben einer grundsätzlich aufgeschlossenen Haltung der beteiligten Organisationen auch eine nicht zu unterschätzende Informationsleistung an die zukünftigen Kunden, die Menschen mit Behinderung, erbracht werden. Um eine aktive und nicht nur scheinbare Einbindung in das System und die Gestaltung des Leistungsbezugs zu verwirklichen und damit den Wandel zum mündigen Kunden tatsächlich zu vollziehen, müssen Rechte und Ansprüche hinsichtlich des Persönlichen Budgets möglichst einfach und gleichzeitig umfassend erklärt werden, damit die Bedeutung des Persönlichen Budgets für die eigene Lebensplanung nachvollzogen und Ziele von den behinderten Menschen selbst formuliert werden können.

Der Martinsclub Bremen hat neben einer ersten Informationsveranstaltung im April 2008 ein monatlich stattfindendes »Budgetcafé« eingerichtet. Hier können Menschen mit Behinderung sowie deren Angehörige zusammenkommen, um sich über das Persönliche Budget zu informieren und gemachte Erfahrungen austauschen. Das Budgetcafé findet im Veranstaltungszentrum des Martinsclubs statt; bei Kaffee und Kuchen werden die Teilnehmenden von Mitarbeiterinnen und Mitarbeitern über ihre Möglichkeiten informiert.

Informationsveranstaltungen und das Budgetcafé sind natürlich nur erste Schritte auf dem Weg zu einer erfolgreichen Implementierung des Persönlichen Budgets. Der Martinsclub Bremen bietet im Herbst 2008 eine Fortbildung zur Budgetbegleiterin und zum Budgetbegleiter an, die zum Beispiel Fachkräfte von Wohneinrichtungen oder gesetzliche Betreuerinnen und Betreuer qualifiziert, als unabhängige Beratungsund Begleitungsfachkraft hinsichtlich des Persönlichen Budgets tätig zu werden.

Mit den Maßnahmen zum Persönlichen Budget stößt der Martinsclub Bremen an personelle und finanzielle Grenzen. Um eine qualitativ hochwertige Beratung und Begleitung mit zunehmenden Ansprüchen gewährleisten zu können, werden zusätzliche finanzielle Mittel benötigt, um die Leistungen zum Persönlichen Budget ausbauen zu können. Es besteht beispielsweise die Idee, ein »Budgetbüro« mit zusätzlichen Mitarbeiterinnen und Mitarbeitern zu eröffnen, die neben Ihrer Beratungstätigkeit auch Ehrenamtliche für die Unterstützung bei der Inanspruchnahme des Persönlichen Budgets an behinderte Menschen vermitteln können.

\section{Simon Brukner}

